\title{
VARIAÇÃO NA RESISTÊNCIA TÊNSIL DE AGREGADOS EM FUNÇÃO DO CONTEÚDO DE CARBONO EM DOIS SOLOS NA REGIÃO DOS CAMPOS GERAIS ${ }^{(1)}$
}

\author{
Ademir de Oliveira Ferreira ${ }^{(2)}$, João Carlos de Moraes Sá( ${ }^{(3)}$, Neyde Fabíola \\ Balarezo Giarola $^{(3)}$, Mônica Gabrielle Harms ${ }^{(4)}$, Simone Miara ${ }^{(5)}$, Marina \\ Araujo Bavoso $^{(6)}$, Clever Briedis ${ }^{(7)}$ \& Caio Quadros Netto ${ }^{(8)}$
}

\begin{abstract}
RESUMO
O comportamento em macroescala do solo depende das propriedades mecânicas de agregados individuais. A resistência tênsil (RT) de agregados do solo é uma das propriedades mecânicas mais úteis e utilizadas como indicadora do impacto do manejo na qualidade do solo. O objetivo deste trabalho foi avaliar o comportamento da $\mathrm{RT}$ em relação ao conteúdo do C orgânico total (COT) em dois solos (Latossolo Vermelho distrófico típico) com classes texturais diferentes, em sistema plantio direto de longa duração. $O$ delineamento experimental utilizado foi fatorial $2 \times 2 \times 2$ inteiramente casualizado, com 12 tratamentos. Os fatores analisados foram: (a) dois solos: Latossolo Vermelho distrófico típico classe textural franco-argiloarenosa e Latossolo Vermelho distrófico típico classe textural francoargilosa; (b) profundidades de amostragem: 0-5 e 5-20 cm; e (c) épocas de amostragem ( $E_{1}$-outubro de 2007; $E_{2}$-setembro de 2008). Para determinação da resistência tênsil, foram coletados 1.440 blocos de solo. A RT e o conteúdo de COT foram mensurados em 1.920 agregados para cada época de amostragem. A RT reduziu à medida que o conteúdo de COT aumentou e com maior impacto no LV classe textural franco-argilosa, ou seja, o conteúdo de argila influenciou o comportamento da RT, que respondeu inversamente proporcional ao conteúdo de argila. O impacto do conteúdo de COT na RT foi mais importante na camada superficial do solo de $0-5 \mathrm{~cm}$ do que na de $5-20 \mathrm{~cm}$.
\end{abstract}

Termos de indexação: resistência tênsil, argila, carbono orgânico do solo.

\footnotetext{
(1) Recebido para publicação em julho de 2010 e aprovado em janeiro de 2011.

(2) Doutorando em Ciência do Solo, Departamento de Manejo e Conservação do Solo. Universidade Federal de Santa Maria UFSM. Av. Roraima 1000, Camobi, CEP 97105900 Santa Maria (RS). Bolsista do CNPq. E-mail: aoferreira1@yahoo.com.br

(3) Professor Adjunto, Departamento de Solos e Engenharia Agrícola, Universidade Estadual de Ponta Grossa - UEPG. Av. Gal. Carlos Cavalcanti 4748, CEP 84030-900 Ponta Grossa (PR). E-mails: jcmsa@uepg.br; neydef@uepg.br

(4) Engenheira Agrônoma, Mestranda em Agronomia, UEPG. E-mail: monicaharms@bol.com.br

(5) Engenheira Agrônoma, UEPG. E-mail: monemiara@yahoo.com.br

(6) Engenheira Agrônoma, Mestranda em Solos e Nutrição de Plantas. Departamento de Ciência do Solo, Escola Superior de Agricultura "Luiz de Queiroz" - ESALQ/USP. Caixa Postal 09, Av. Pádua Dias 11, CEP 13418-900 Piracicaba (SP). Bolsista do CNPq. E-mail: marinabavoso@hotmail.com

(7) Engenheiro Agrônomo, Mestre em Agronomia, UEPG. E-mail: cleverbriedis@yahoo.com.br

${ }^{(8)}$ Acadêmico do curso de Agronomia, UEPG. E-mail: caio-quadros@hotmail.com
} 


\title{
SUMMARY: VARIATION IN AGGREGATE-TENSILE STRENGTH AS A FUNCTION OF CARBON CONTENT OF TWO SOILS IN THE REGION OF CAMPOS GERAIS
}

\begin{abstract}
Macroscale soil behavior depends on the mechanical properties of individual aggregates. Tensile strength (TS) of soil aggregates has been used as an indicator of management effect on soil quality as related to land use. The objective of this study was to evaluate the change of TS in relation to the variation of soil organic carbon (SOC) content in two soils (Typic Hapludox) with different textures under a long term no-tillage system. The experiment was arranged in a completely randomized $2 \times 2 \times 2$ factorial design with 12 treatments. The analyzed factors were: (a) two soils: Oxisol with medium texture and Oxisol with clay loam texture, (b) two sampling layers: 0-5 and 5-20 cm, (c) two sampling dates (October 2007-T, September 2008$\left.T_{2}\right)$. To determine TS, 1440 soil blocks were sampled. The TS and SOC contents were measured in 1920 aggregates for each sampling time. RT decreased as SOC content increased, with a greater impact on Oxisol with clay loam texture. The clay content affected the TS behavior, to which it was inversely proportional. The impact of SOC content on TS was greater in the soil surface $(0-5 \mathrm{~cm})$ than in the 5-20 cm layer.
\end{abstract}

Index terms: tensile strength, clay, soil organic carbon.

\section{INTRODUÇÃO}

O comportamento em macroescala do solo depende das propriedades mecânicas de agregados individuais. A resistência tênsil (RT) de agregados do solo é utilizada como indicadora do impacto do manejo na qualidade do solo, em resposta aos processos físicos e mecânicos que ocorrem com o uso da terra. A RT é definida como o estresse ou força por unidade de área requerida para fraturar os agregados do solo, quando submetidos a uma pressão (Dexter \& Watts, 2000). Esse parâmetro é influenciado por diversos fatores, como a umidade do solo (Utomo \& Dexter, 1981), o conteúdo de argila dispersa no solo, os ciclos de umedecimento e secagem, o teor de matéria orgânica do solo (Dexter \& Watts, 2000), o teor e a mineralogia da fração argila (Kemper et al., 1987) e os materiais cimentantes (Kay \& Dexter, 1992), além das condições climáticas, práticas de manejo e características do solo (Kay \& Dexter, 1992). Em razão disso, a RT dos agregados tem sido proposta como uma medida sensível para avaliar o efeito na estrutura do solo dos sistemas de manejo (Watts \& Dexter, 1997; Munkholm et al., 2001; Munkholm \& Schjønning, 2004; Blanco-Canqui et al., 2005).

Bavoso et al. (2010), na região dos Campos Gerais, estudaram o efeito de diferentes sistemas de preparo do solo na resistência tênsil de agregados e concluíram que as alterações macroestruturais provocadas por esses sistemas promoveram modificações na RT; esses autores afirmaram que isso ocorreu em razão do pouco ou não significativo acúmulo de matéria orgânica do solo (MOS) entre esses sistemas.

A MOS contribui para o aumento dos valores de RT quando o teor de argila+silte é elevado (Imhoff, 2002). Em decorrência disso, especialmente em Latossolos, o sistema plantio direto (SPD) de longa duração, aliado ao fluxo contínuo de carbono oriundo da decomposição de resíduos culturais, tem tido grande importância na contribuição da proteção física da MOS (Sá et al., 2001, 2008). O SPD com elevado aporte de resíduos, associado ao acréscimo de carbono, pode causar diminuição na RT na camada superficial do solo devido à diluição da MOS (Favoretto et al., 2008).

Vários trabalhos relacionaram o conteúdo de carbono (C) com a RT. Chan (1995) e Tormena et al. (2008a) observaram correlação linear significativa entre resistência tênsil de agregados e teores de $\mathrm{C}$ orgânico do solo em Latossolo de textura média, indicando que a resistência tênsil de agregados aumentou proporcionalmente com a redução dos teores de C orgânico do solo. Blanco-Canqui et al. (2005) também obtiveram correlação exponencial significativa entre esses parâmetros, porém em Argissolo, mostrando que o aumento de C orgânico do solo implica redução da resistência tênsil do solo.

Também em Latossolos da região subtropical, Bartoli et al. (1992) e Guimarães et al. (2009) reportaram efeito contrário, ou seja, o aumento no conteúdo de C orgânico total (COT) foi proporcional ao maior valor de RT do solo. Segundo esses autores, esse efeito deve-se à cimentação entre as partículas minerais de argila e a matéria orgânica mais humificada do solo.

No entanto, essa correlação significativa entre o conteúdo de $\mathrm{C}$ e a resistência tênsil não foi verificada em trabalhos de Imhoff et al. (2002) e Tormena et al. (2008b). Já Watts \& Dexter (1998), em virtude da utilização de solos com faixa mais ampla de teor de $\mathrm{C}$ orgânico, obtiveram correlação significativa entre esses parâmetros.

O objetivo deste trabalho foi avaliar o comportamento da RT em relação ao conteúdo do C orgânico total (COT) em dois solos com classes texturais diferentes, em sistema plantio direto de longa duração. 


\section{MATERIAL E MÉTODOS}

\section{Localização, clima e descrição da área de estudo}

O presente trabalho foi desenvolvido na Fazenda Escola Capão da Onça - FESCON, situada a 990 m de altitude, sob as coordenadas geográficas de $25^{\circ} 05^{\prime} 49$ " LS e $50^{\circ} 03^{\prime} 11^{\prime \prime} \mathrm{LW}$.

A área experimental foi situada em uma topossequência abrangendo solos profundos e bem drenados nos terços superior e médio da vertente, enquanto no terço inferior os solos possuem drenagem mais impedida. Os solos analisados foram: Latossolo Vermelho distrófico típico, com classe textural francoargiloarenosa, e Latossolo Vermelho distrófico típico, com classe textural franco-argilosa (Figura 1). O material de origem foi derivado de material retrabalhado de arenitos da formação Furnas e folhelhos da formação Ponta Grossa. A vegetação nativa regional é constituída por composição florística denominada campos subtropicais dos Campos GeraisPR. O relevo é suave ondulado, com pendentes entre 5 e $7 \%$ de declividade.

O clima é caracterizado como subtropical úmido, mesotérmico, do tipo Cfb (classificação de Köppen). No ano do experimento (outubro de 2007 a setembro 2008) a temperatura média máxima foi de $26{ }^{\circ} \mathrm{C}$ e a mínima de $13^{\circ} \mathrm{C}$ (Quadro 1), e a pluviosidade total foi de $1.558 \mathrm{~mm}$ (IAPAR, 2008).

Este estudo foi conduzido em uma área com predominância de Latossolo Vermelho há longo período sob plantio direto (20 anos) com rotação de culturas: trigo/soja/aveia-preta + ervilhaca/milho. A quantidade de resíduos culturais das áreas em estudo foi, em média, de 5,16 $\mathrm{Mg}_{\mathrm{ha}}{ }^{-1}$.

\section{Características morfológicas}

Do ponto de vista da morfologia, os solos estudados apresentam sequência de horizontes A-Bw-C. O horizonte $\mathrm{C}$ é profundo em ambos os solos (abaixo de $250 \mathrm{~cm}$ ) e constituído de arenito. Nesses solos há pouco incremento de argila com a profundidade e observa-se uma transição difusa ou gradual entre os horizontes; apresentam estrutura do tipo moderada e forte em blocos subangulares e angulares.

\section{Latossolo Vermelho distrófico típico, com classe textural franco-argilo arenosa}

- Horizonte A moderado (profundidade de 0 a $30 \mathrm{~cm}$ ). A coloração é bruno avermelhada escura no matiz 5YR 3/6 (seco). Apresenta estrutura em blocos subangulares, grossa e forte, porosidade elevada, consistência dura, friável, não plástica, cerosidade ausente.

- Horizonte B latossólico (profundidade de 31 a $250 \mathrm{~cm}$ ). A coloração é bruno avermelhada escura e vermelhoescura no matiz 2,5YR 3/6 (seco). Apresenta estrutura em blocos subangulares, grossa e forte, porosidade elevada, consistência dura, friável, não plástica, cerosidade ausente.
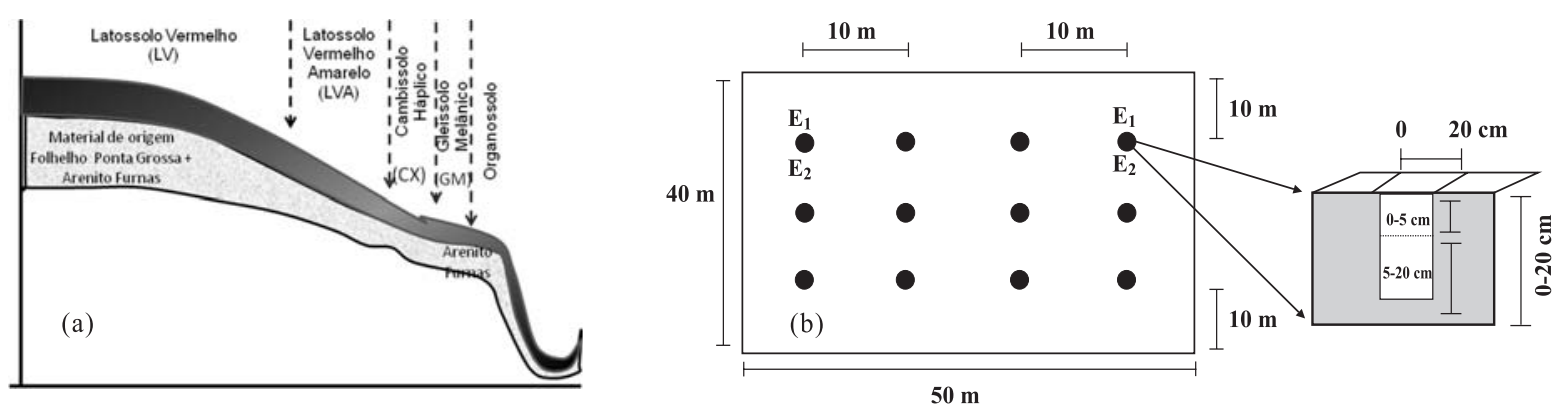

Figura 1. (a) Perfil topográfico da pedossequência; (b) croqui do bloco com o detalhe da localização dos tratamentos e do procedimento de amostragem do solo. Fonte: Ferreira (2009).

Quadro 1. Distribuição mensal da precipitação pluvial

\begin{tabular}{|c|c|c|c|c|c|c|c|c|c|c|c|c|c|c|}
\hline Descrição & & & & & & $\mathbf{M}$ & ses & & & & & & & Média \\
\hline \multirow{3}{*}{$\operatorname{Dados}^{(1)}$} & \multirow{3}{*}{ Precip } & Jan & Fev & Mar & Abr & Maio & Jun & Jul & Ago & Set & Out & Nov & Dez & \multirow{3}{*}{119,1} \\
\hline & & 188 & 155 & 136 & 105 & 118 & 115 & 95 & 80 & 134 & 149 & 120 & 150 & \\
\hline & & Out & Nov & Dez & Jan & Fev & Mar & Abr & Maio & Jun & Jul & Ago & Set & \\
\hline $2007-2008^{(2)}$ & Precip & 60 & 195 & 214 & 154 & 158 & 105 & 161 & 83 & 137 & 54 & 184 & 53 & 129,8 \\
\hline
\end{tabular}

${ }^{(1)}$ Dados históricos em Ponta Grossa no período de 44 anos para os meses de janeiro a dezembro. ${ }^{(2)}$ Dados do experimento no período de outubro de 2007 a setembro de 2008 . 
Latossolo Vermelho distrófico típico, com classe textural franco-argilosa

- Horizonte A moderado (profundidade de 0 a $40 \mathrm{~cm}$ ). A coloração é bruno avermelhada escura no matiz 2,5YR 3/4 (seco). Apresenta estrutura em blocos angulares e subangulares e é friável, ligeiramente plástico e ligeiramente pegajoso.

- Horizonte B latossólico (profundidade de 41 a $250 \mathrm{~cm}$ ). A coloração é bruno avermelhada escura e vermelhoescura no matiz 2,5YR 3/6 (seco). Apresenta estrutura em blocos angulares e subangulares, porosidade elevada e consistência macia, friável, ligeiramente plástico e ligeiramente pegajoso.

\section{Delineamento experimental}

$\mathrm{O}$ delineamento experimental utilizado foi um arranjo em fatorial $2 \times 2 \times 2$ inteiramente casualizado, com 12 tratamentos. Os fatores considerados foram: (a) Solos: Latossolo Vermelho distrófico típico, com classe textural franco-argiloarenosa - TM (232,5 g kg-1 de argila); Latossolo Vermelho distrófico típico, com classe textural franco-argilosa - TA $\left(401 \mathrm{~g} \mathrm{~kg}^{-1} \mathrm{de}\right.$ argila); (b) Profundidades de amostragem: 0-5 e 5$20 \mathrm{~cm}$; (c) epocas de amostragem: época $1\left(E_{1}\right)$, após colheita do trigo (outubro de 2007 ); época $2\left(E_{2}\right)$, após o manejo mecânico (rolagem com rolo-faca) da aveiapreta + ervilhaca (setembro de 2008).

Os tratamentos foram dispostos no campo na forma de transectos, conforme detalhes apresentados no croqui do experimento no campo (Figura 1).

\section{Análise granulométrica e química}

A análise granulométrica foi realizada utilizando o método de Bouyoucos, conforme descrito por Embrapa (1997).

As análises químicas foram realizadas conforme Pavan et al. (1992), em amostras compostas (10 subamostras) nas profundidades de $0-5$ e $5-20 \mathrm{~cm}$ (Quadro 2).

\section{Determinação de C orgânico total - COT}

$\mathrm{O}$ conteúdo de $\mathrm{C}$ nas classes de agregados e na fração particulada foi determinado pelo método da combustão seca, utilizando um determinador elementar de C e N (TruSpec CN LECO ${ }^{\circledR} 2006$, St. Joseph, EUA).

\section{Cálculo da resistência tênsil - RT}

A resistência tênsil foi calculada conforme descrito por Dexter \& Kroesbergen (1985):

$$
\mathrm{RT}=0,576\left(\mathrm{P} / \mathrm{D}^{2}\right)
$$

em que RT é a resistência tênsil; 0,576, o coeficiente de proporcionalidade, resultante da relação entre a carga compressiva aplicada e o estresse tênsil gerado no interior do agregado; $\mathrm{P}$, a força necessária para a quebra tênsil do agregado $(\mathrm{N})$; e D, o diâmetro efetivo (m). O diâmetro efetivo (D) foi calculado conforme Watts \& Dexter (1998):

$$
\mathrm{D}=\operatorname{Dm}\left(\mathrm{M} / \mathrm{M}_{0}\right)^{1 / 3}
$$

em que M é a massa do agregado individual (g); $\mathrm{M}_{0}$, a massa média dos agregados na população (g); e Dm, o diâmetro médio dos agregados $(\mathrm{mm})$, definido pela média dos tamanhos das peneiras $[(12,5+19,0) / 2=$ $15,75 \mathrm{~mm}$ ]. Usualmente, a medida da resistência tênsil dos agregados de solo é expressa em $\mathrm{kPa}$.

$\mathrm{O}$ equipamento utilizado foi um dinamômetroIMPAC, marca Lutron, modelo FG-20 KG (Figura 2).

Os resultados foram submetidos a análise pelo programa SISVAR 5.0, utilizando o teste de Tukey a $5 \%$. Para obtenção das curvas de resposta, foi utilizado o procedimento da análise de regressão pelo programa JMP IN versão 3.2.1 (Sall et al., 2005), utilizando o teste $\mathrm{F}$ a 5,1 e 0,1 \% de probabilidade.

\section{RESULTADOS E DISCUSSÃO}

As variáveis C orgânico total (COT), RT, conteúdo de argila e densidade do solo apresentaram interação significativa com a classe textural, a profundidade e a época de amostragem (Quadro 3).

O aumento no conteúdo de COT (média geral dos tratamentos) resultou na diminuição da RT e teve

Quadro 2. Caracterização química e física do Latossolo Vermelho textura média (TM) e textura argilosa (TA)

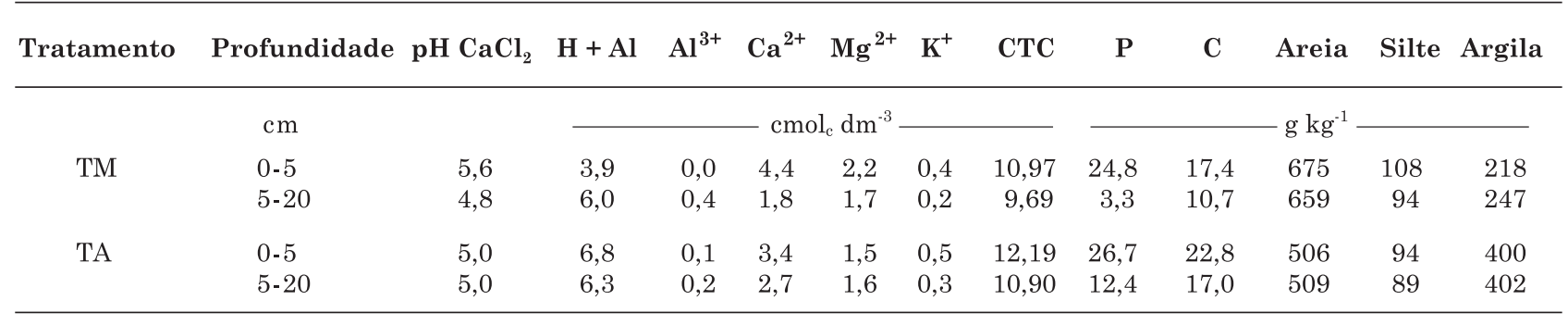



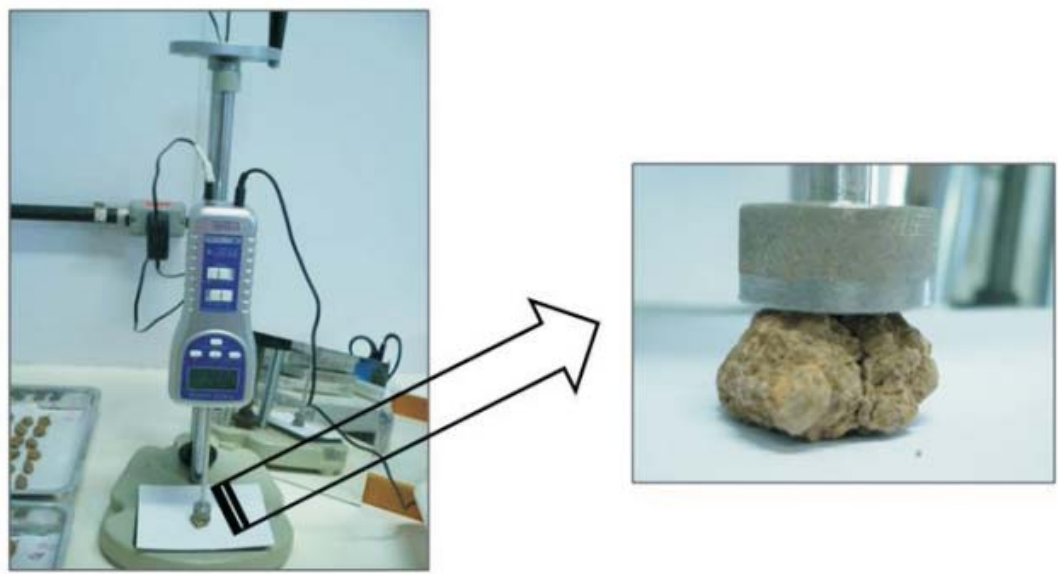

Figura 2. Dinamômetro, com detalhes da fratura do agregado. Fonte: Ferreira (2009).

relação linear e significativa $\left(\mathrm{R}^{2}=0,68 ; \mathrm{p}=0,0009\right)$ entre esses dois parâmetros (Figura 3). Isso ocorreu em razão do aumento da fração lábil de MOS (compostos alifáticos com menor força de ligação). Além disso, os macroagregados apresentam a maior presença de agentes temporários, como raízes e hifas de fungos, que contribuem para o aumento da porosidade interna dos agregados (Tisdall \& Oades, 1982). Outro fator que explica esse efeito é o teor de alumínio desse solo (32\% de gibbsita), ou seja, Ferreira et al. (1999) confirmam que Latossolos gibbsíticos apresentaram menor densidade do solo, menor estabilidade de agregados em água, menor macroporosidade e menor permeabilidade, quando comparados a Latossolos cauliníticos. Isso porque a presença dos agentes temporários de agregação (Tisdall \& Oades, 1982) propicia maior entrada de COT e, em consequência, pode ter reduzido a força entre as partículas, por causa da ação dos compostos alifáticos ao formarem ligações mais fracas com a superfície dos minerais de argila. Assim, esses compostos irão atuar com menor força de ligação, resultando em menor $\mathrm{RT}$.

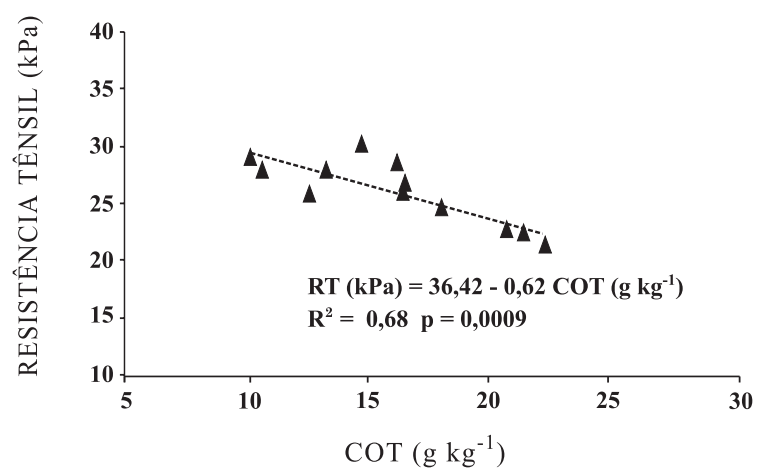

Figura 3. Regressão linear entre o conteúdo de carbono orgânico total (COT) e a resistência tênsil (RT) nos agregados de um Latossolo Vermelho em plantio direto de longa duração. A regressão foi realizada com a média geral dos 12 tratamentos e das duas classes texturais.
A relação entre o COT e a $\mathrm{RT}$ foi ainda mais evidente quando observada comparativamente nas duas profundidades de amostragem. Nesse caso, a regressão linear entre essas duas variáveis foi significativa apenas na camada de 0-5 cm (Quadro 4). Esse contraste entre as camadas pode ser explicado pelo maior conteúdo de COT na camada superficial, em decorrência da contínua adição de resíduos culturais na superfície do solo (Sá \& Lal, 2009). Ou seja, o fluxo contínuo de resíduos de culturas na superfície do solo aumentou o COT, contribuindo para o C orgânico gradiente através das camadas (Tormena et al., 2004).

No sistema plantio direto, o não revolvimento do solo, a rotação de culturas e a manutenção dos resíduos culturais na superfície do solo proporcionam a decomposição lenta e gradual do material vegetal depositado, o que, associado com a fração mineral do solo, favorece o acúmulo da MOS (SÁ et al., 2001) e estimula a formação e a estabilidade de agregados (Tisdall \& Oades, 1982; Six et al., 2002, 2006).

Guimarães et al. (2009) notaram que uma planta de sistema radicular agressivo e crescimento contínuo, aliada à decomposição, pode contribuir para uma diminuição da resistência tênsil no solo. Nesse caso, fica evidente que o SPD bem feito, com elevado aporte de resíduos, associado à rotação de culturas com espécies de diversificado sistema radicular são fatores que reduzem a RT. Esses efeitos benéficos da rotação de culturas nas propriedades físicas dos solos têm sido demonstrados pela redução na densidade do solo e aumento na porosidade (Albuquerque et al., 1995), bem como pelo controle da temperatura do solo e aumento na disponibilidade de água (Derpsch et al., 1985).

No presente estudo, outro fator que alterou diretamente a $\mathrm{RT}$ foi o aporte de resíduos culturais (5,16 $\left.\mathrm{Mg} \mathrm{ha}^{-1}\right)$ na superficie do solo, ou seja, esse comportamento ocorreu em função do significativo aporte de matéria orgânica fresca oriunda da decomposição desses resíduos na superfície, causando 
Quadro 3. ANOVA do experimento

\begin{tabular}{llll}
\hline Causa da variação & COT & RT & Argila \\
\hline & $\mathrm{g} \mathrm{kg}^{-1}$ & $\mathrm{kPa}$ & $\%$ \\
Textura (T) & $*$ & $*$ & $*$ \\
Profundidade (P) & $*$ & $*$ & $*$ \\
Época (E) & $*$ & $*$ & $*$ \\
CV (\%) & 3,54 & 6,87 & 5,22 \\
T x P & $*$ & $*$ & $*$ \\
T x E & $*$ & $*$ & $*$ \\
P x E & $*$ & $*$ & $*$ \\
T x P x E & $*$ & $*$ & $*$ \\
CV (\%) & 2,84 & 2,41 & 1,61 \\
\hline *: significativo a $5 \%$. & & &
\end{tabular}

diluição da matéria orgânica mais humificada (Favoretto et al., 2008), diminuindo dessa forma a RT.

Zhang (1994) citado por Tormena (2008a) indica que há dois efeitos contrários da matéria orgânica do solo sobre a resistência tênsil dos agregados: 0 aumento no número e na força das ligações entre as partículas; e o efeito de diluição, que implica redução da densidade do solo ou aumento da porosidade do agregado. Além da quantidade de matéria orgânica do solo, ressaltase que o seu grau de humificação influencia a resistência tênsil dos agregados, conforme reportado por Zhang (1994), demonstrando que, quanto mais humificada a matéria orgânica do solo, menor será o seu efeito em reduzir a resistência tênsil dos agregados. Resultados semelhantes foram obtidos por BlancoCanqui et al. (2005) em Ultisol (Argissolo) e Chan (1995) e Tormena et al. (2008a) em Oxisol (Latossolo) com textura média. Por outro lado, os resultados reportados por Bartoli et al. (1992), Imhoff et al. (2002) e Tormena et al. (2008b) contrastam com os anteriores, nos quais os dois últimos autores não obtiveram relação positiva entre RT e COT em razão do uso de uma faixa mais restrita de teor de C. Bartoli et al. (1992) e Guimarães et al. (2009), trabalhando com Latossolos situados na região tropical e subtropical, reportaram aumento no conteúdo de COT proporcional ao maior valor de RT do solo e afirmaram que esse efeito é consequência da cimentação entre as partículas minerais de argila e a matéria orgânica mais humificada do solo. Ou seja, a fração argila, por apresentar elevada reatividade devido à presença de cargas elétricas, favorece, assim, a formação de ligações entre partículas minerais e, ou, partículas minerais e orgânicas (Imhoff, 2002).

Na região de Ponta Grossa-PR, Favoretto et al. (2008) encontraram em um Latossolo Vermelho distrófico sob plantio direto maior conteúdo de $\mathrm{C}$ na camada de 0-20 cm do que o encontrado neste estudo. Todavia, constataram que o grau de humificação da matéria orgânica avaliado pelo método da fluorescência induzida a laser (HFIL) era baixo. Esses autores explicaram que esse comportamento ocorre em razão do aporte expressivo de resíduos orgânicos frescos na superfície do solo, que, durante o processo de decomposição, causa a diluição da matéria orgânica mais humificada. Assim, mesmo solos com elevado conteúdo de $\mathrm{C}$ podem ter grau de humificação da matéria orgânica mais baixo do que o de solos com menor conteúdo de C. No sistema plantio direto, a preservação das estruturas mais lábeis (por exemplo, compostos alifáticos constituídos por polissacarídeos e proteínas interestratificadas com polissacarídeos) nos agregados é muito importante porque ocorre a maior proteção física intra-agregado, tornando-se um dos pontos-chave para explicar o acúmulo de C nesse sistema de manejo (Bayer et al., 2001; Sá et al., 2001; Amado et al., 2006; Zotarelli et al., 2007; Sá et al., 2008; Diekow et al., 2009).

A regressão linear entre o conteúdo de COT e a RT (Quadro 4) para o LV textura argilosa foi significativa $\left(\mathrm{R}^{2}=0,93 ; \mathrm{p}=0,002\right)$ e superior à do $\mathrm{LV}$ textura média $\left(R^{2}=0,06 ; p=0,64\right)$.

Essa influência no comportamento da RT evidencia a importância da textura do solo no estudo desse atributo, principalmente porque a causa está relacionada com as características físicas e químicas do tipo de mineral de argila e não somente com o conteúdo desse mineral. Os resultados reportados por Imhoff et al. (2002) com a utilização de regressão múltipla foram a influência da textura e do C orgânico sobre a RT e o fato de que os teores de argila+silte e MOS foram as variáveis que mais influenciaram a $\mathrm{RT}$, havendo interação significativa entre as frações mineral e orgânica do solo.

Os resultados obtidos por Tormena et al. (2008a) mostraram relação significativa entre a textura e a RT em um LV textura média, contrastando com os deste estudo. Entretanto, há duas diferenças importantes: as argilas citadas no trabalho de Tormena et al. (2008a) são oriundas de rochas basálticas e possuem conteúdo mais elevado de ferro (Pierangeli et al., 2005) - a influência do ferro na microagregação e macroagregação de Latossolos é bem conhecida e descrita na literatura; e o tempo de adoção do plantio direto e os aportes de resíduos culturais são inferiores aos citados neste estudo.

Bavoso et al. (2010) observaram que o SPD apresentou RT cerca de $60 \%$ maior que a dos preparos convencionais e entre 44 e $18 \%$ maior que a dos sistemas com mínima mobilização, sugerindo um gradiente de RT com redução dos seus valores à medida que aumentou a intensidade de mobilização do solo. Segundo esses autores, a mobilização do solo alterou a estrutura por meio da quebra nas ligações entre agregados e expôs o solo a secagem e umedecimento, contribuindo para os mecanismos que promoveram o arranjo de fendas e microfendas, definindo as zonas de fraqueza na estrutura do solo. No entanto, os mesmos autores não constataram diferenças significativas no teor de MOS entre os sistemas de uso e manejo, provavelmente por se tratar de um experimento de apenas três anos de implantação. 
Bavoso et al. (2010) também observaram em SPD de três anos um valor de RT elevado $(51,72 \mathrm{kPa})$, o que ocorreu porque o SPD estava na fase inicial, em que o baixo acúmulo de resíduos e o rearranjo da estrutura do solo são as principais características (Sá et al., 2004). No presente estudo, em SPD de longa duração (20 anos) com maior acúmulo de resíduos associado ao acréscimo de carbono, observou-se diminuição na RT devido à diluição da MOS (Favoretto et al., 2008) causada pela decomposição do elevado aporte desses resíduos.

\section{Resistência tênsil de agregados alterada pela classe textural}

A média geral da RT (considerando as camadas de 0-5 e 5-20 cm) para o LV textura média foi de $28,3 \mathrm{kPa}$, enquanto para o LV textura argilosa foi de $24,5 \mathrm{kPa}$. Para o conteúdo de COT, a média geral do LV textura argilosa foi de $19,3 \mathrm{~g} \mathrm{~kg}^{-1}$, enquanto no $\mathrm{LV}$ textura média foi de $13,0 \mathrm{~g} \mathrm{~kg}^{-1}$. Essa diferença no conteúdo de COT representa 48,0 \% mais C no LV textura argilosa. A diferença da RT entre as texturas foi ainda mais acentuada ao se considerar a camada de $0-5 \mathrm{~cm}$. Nesse caso, a média do COT para o LV textura média foi de $14,9 \mathrm{~g} \mathrm{~kg}^{-1} \mathrm{e}$ a RT, de $28,5 \mathrm{kPa}$, ao passo que no LV textura argilosa a média do COT foi de $21,6 \mathrm{~g} \mathrm{~kg}^{-1} \mathrm{e}$ a RT, de $22,2 \mathrm{kPa}$.

A relação entre a $\mathrm{RT}$ e o conteúdo de argila foi linear e significativa $\left(R^{2}=0,54 ; p=0,006\right)$, indicando que a diminuição da RT está em parte associada ao incremento do conteúdo de argila nos Latossolos em estudo (Figura 4).

A relação do conteúdo de argila com a RT seguiu a mesma tendência observada para o COT com a profundidade de amostragem, ao ocorrer relação linear e significativa no LV textura argilosa em comparação ao LV textura média (Quadro 4). Os resultados reportados por Imhoff et al. (2002) evidenciam que a argila+silte e a MOS foram as variáveis que mais influenciaram a RT. Mostraram também a variação

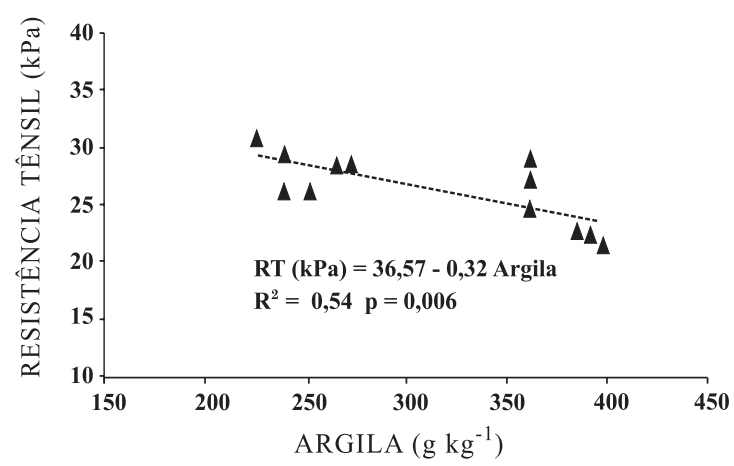

Figura 4. Regressão entre o conteúdo de argila e a resistência tênsil (RT) nos agregados de um Latossolo Vermelho sob plantio direto de longa duração. Os valores utilizados para a equação de regressão foram obtidos da média geral do LV nas duas texturas e nas duas profundidades amostradas.
Quadro 4. Equações de regressão entre o conteúdo de carbono orgânico total (COT) e a resistência tênsil (RT) e entre o conteúdo de argila e a resistência tênsil (RT), nos agregados de um Latossolo Vermelho em plantio direto de longa duração

\begin{tabular}{clll}
\hline \multirow{2}{*}{ Profundidade } & \multicolumn{1}{c}{ Equação } & $\mathbf{R}^{2}$ & NS (P) \\
\hline \multirow{2}{*}{$0 \mathrm{(1)}$} & & & \\
$0-5$ & $\mathrm{RT} \S=42,26-0,93$ COT $\S$ & 0,88 & 0,005 \\
& $\mathrm{RT}=39,7-0,45$ Argila $\$ \S$ & 0,90 & 0,004 \\
$5-20$ & $\mathrm{RT}=31,93-0,32$ COT & 0,34 & 0,23 \\
Textura & $\mathrm{RT}=30,07-0,08$ Argila & 0,08 & 0,57 \\
Média & $\mathrm{RT}=30,7-0,19$ COT & 0,06 & 0,64 \\
& $\mathrm{RT}=36,19-0,32$ Argila & 0,09 & 0,55 \\
Argilosa & $\mathrm{RT}=45,54-1,09$ COT & 0,93 & 0,002 \\
& $\mathrm{RT}=82,9-1,54$ Argila & 0,78 & 0,02 \\
\hline
\end{tabular}

(1) NS (P): nível de significância da regressão. ${ }^{\S} \mathrm{RT}$ : kPa; ${ }^{\S \S}$ COT: $\mathrm{g} \mathrm{kg}^{-1}$; $\$$ Argila: $\mathrm{g} \mathrm{kg}^{-1}$.

da resistência tênsil em função do teor de argila+silte, para diferentes níveis de MO (20,78; 27; e $\left.32 \mathrm{~g} \mathrm{~kg}^{-1}\right)$.

A profundidade de amostragem influenciou o comportamento da RT em relação ao conteúdo de argila. Na camada superficial de $0-5 \mathrm{~cm}$ a relação linear entre o conteúdo de argila e a RT foi elevada e significativa, enquanto na camada de 5-20 cm não houve relação entre ambas (Quadro 4).

\section{CONCLUSÕES}

1. A resistência tênsil (RT) reduziu à medida que o conteúdo de COT aumentou, com maior impacto no LV textura argilosa nas condições estudadas.

2. A RT foi maior com o aumento da profundidade de amostragem.

3. Na camada superficial de $0-5 \mathrm{~cm}$ observou-se relação linear inversa entre o conteúdo de argila e a RT, o mesmo não acontecendo para a camada de 5$20 \mathrm{~cm}$.

\section{AGRADECIMENTOS}

À Agrisus (Agricultura Sustentável) e ao Laboratório de Matéria Orgânica do Solo (Labmos UEPG), pelo auxílio financeiro na condução do experimento.

\section{LITERATURA CITADA}

ALBUQUERQUE, J.A.; REINERT, D.J.; FIORIN, J.E.; RUEDELL, J.; PETRERE, C. \& FONTINELLI, F. Rotação de culturas e sistemas de manejo do solo: Efeito sobre a forma da estrutura do solo ao final de sete anos. R. Bras. Ci. Solo, 19:115-119, 1995. 
AMADO, T.J.C.; BAYER, C.; CONCEIÇÃO, P.C.; SPAGNOLLO, E.; CAMPOS, B-H.C. \& VEIGA, M. Potential of carbon acumulation in no-till soils with intensive use and cover crops in Southern Brazil. J. Environ. Qual., 35:1599-1607, 2006.

BLANCO-CANQUI, H.; LAL, R.; OWENS, L.B.; POST, W.M. \& IZAURRALDE, R.C. Mechanical properties and organic carbon of soil aggregates in the Northern Appalachians. Soil Sci. Soc. Am. J., 69:1472-1481, 2005.

BARTOLI, F.; BURTIN, G. \& GUÉRIF, J. Influence of organic matter on aggregation in Oxisols rich in gibbsite or in goethite. II. Clay dispersion, aggregate strength and water-stability. Geoderma, 54:259-274, 1992.

BAYER, C.; MARTIN-NETO, L.; MIELNICZUK, J.; PILLON, C.N. \& SANGOI, L. Changes in soil organic matter fractions under subtropical no-till cropping systems. Soil Sci. Soc. Am. J., 65:1473-1478, 2001.

BAVOSO, M.A.; GIAROLA, N.F.B.; FABIOLA, N.; TORMENA, C.A. \& PAULETTI, V. Preparo do solo em áreas de produção de grãos, silagem e pastejo: Efeito na resistência tênsil e friabilidade de agregados. R. Bras. Ci. Solo, 34:227 234, 2010.

CHAN, K.Y. Strength characteristics of a potentially hardsetting soil under pasture and conventional tillage in the semi-arid region of Australia. Soil Tillage Res. 34:105-113, 1995.

DERPSCH, R.; SIDIRAS, N. \& HEINZMANN, F.X. Manejo do solo com coberturas verdes de inverno. Pesq. Agropec. Bras., 20:761-773, 1985 .

DIECKOW, J.; BAYER, C.; CONCEICÃO, P.C.; ZANATTA, J.A.; MARTIN-NETO, L.; MILORI, D.B.M.; SALTON, J.C.; MACEDO, M.M.; MIELNICZUK, J. \& HERNANI, L.C. Land use, tillage, texture and organic matter stock and composition in tropical and subtropical Brazilian soils. Eur. J. Soil Sci., 60:240-249, 2009.

DEXTER, A.R. \& KROESBERGEN, B. Methodology for determination of tensile strength of soil aggregates. J. Agric. Eng. Res., 31:139-147, 1985.

DEXTER, A.R. \& WATTS, C. Tensile strenght and friability. In: SMITH, K. \& MULLins, C., eds. Soil and environmental analysis: Physical methods. 2.ed. New York, Marcelo Dekker, 2000. p.401-430.

EMPRESA BRASILEIRA DE PESQUISA AGROPECUÁRIA EMBRAPA. Serviço Nacional de Levantamento e Conservação dos Solos. Manual de métodos de análise de solo. 2.ed. Rio de Janeiro, Ministério da Agricultura, 1997. $212 \mathrm{p}$.

FAVORETTO, C.M.; GONÇLVES, D.; MILORI, D.M.B.P.; ROSA, J.A.; LEITE, W.C.; BRINATTI, A.M. \& SAAB, S.C. Determination of humification degree of organic matter of an oxisol and of its organo-mineral fractions. Química Nova, 31, n.8, 1994-1996, 2008.

FERREIRA, M.M.; FERNANDES, B. \& CURI, N. Influência da mineralogia da fração argila nas propriedades físicas de Latossolos da região sudeste do Brasil. R. Bras. Ci. Solo, 23:515-524, 1999.
FERREIRA, A.O. Compartimentos da matéria orgânica como indicadores do sequestro de carbono em sistema plantio direto de longa duração. Ponta Grossa, Universidade Estadual de Ponta Grossa 2009. 98p. (Tese de Mestrado)

KEMPER, W.D.; ROSENAU, R.C. \& DEXTER, A.R. Cohesion development in disrupted soils as affected bay clay and organic matter content and temperature. Soil Sci. Soc. Am. J., 51:860-867, 1987.

GUIMARÃES, R.M.L.; TORMENA, C.A.; ALVES, S.J.; FIDALSKI, J. \& BLAINSKI, E. Tensile strength, friability and organic carbon in an oxisol under a crop-livestock system. Sci. Agric., 66:499-505, 2009.

KAY, B.D. \& DEXTER, A.R. The influence of dispersive clay and wetting/drying cicles on the tensile strength of a redbrown Earth. Austr. J. Soil Res., 30:297-310, 1992.

INSTITUTO AGRONOMICO DO PARANA - IAPAR. Dados climatológicos de Ponta Grossa - 2008. Ponta Grossa, 2008.

IMHOFF, S.; SILVA, A.P. \& DEXTER, A.R. Factors contributing to the tensile strength and friability of Oxisols. Soil Sci. Soc. Am. J., 66:1656-1661, 2002.

MUNKHOLM, L.J.; SCHJØNNING, P. \& RASMUSSEN, K.J. No-inversion tillage effects on soil mechanical properties of a humid sandy loam. Soil Tillage Res., 62:1-14, 2001.

MUNKHOLM, L.J. \& SCHJØNNING, P. Structural vulnerability of a sandy loam exposed to intensive tillage and traffic in wet conditions. Soil Tillage Res., 79:79-85, 2004.

PAVAN, M.A.; BLOCH, M.F.; ZEMPULSKI, H.C.; MIYAZAWA, M. \& ZOCOLER, P.C. Manual de análise química do solo e controle de qualidade. Curitiba, Instituto Agronômico do Paraná, 1992. 38p. (Circular, 76)

PIERANGELI, M.A.P.; GUILHERME, L.R.G.; CURI, N.; SILVA, M.L.N.; LIMA, J.M. \& COSTA, E.T.S. Efeito do pH na adsorção e dessorção de cádmio em Latossolos brasileiros. R. Bras. Ci. Solo, 29:523-532, 2005.

SÁ, J.C.M.; CERRI, C.C.; DICK, A.W.; LAL, R.; VENSKE FILHO, S.P.; PICCOLO, M.C. \& FEIGL, B.E. Soil organic matter dynamics and sequestration rates for a tillage chronosequence in a Brazilian Oxisol. Soil Sci. Soc. Am. J., 65:1476-1499, 2001.

SÁ, J.C.M.; CERRI, C.C.; PICCOLO, M.C.; FEIGL, B.E.; FORNARI, A.; SÁ, M.F.M.; VENZKE FILHO, S.P.; SEGUY, L.; BOUZINAC, S. \& PAULLETI, V. O plantio direto como base do sistema de produção visando o sequestro de carbono. R. Plantio Direto, 84:45-61, 2004.

SÁ, J.C.M. \& LAL, R. Stratification ratio of soil organic matter pools as an indicator of carbon sequestration in a tillage chronosequence on a Brazilian Oxisol. Soil Till. Res, v.103, p.46-56, 2009.

SÁ, J.C.M.; SA, M.F.M.; SANTOS, J.B. \& FERREIRA, A.O. Dinâmica da matéria orgânica nos Campos Gerais.. In: SANTOS, G.A.; SILVA, L.S.; CANELLAS, L.P. \& CAMARGO, F.A.O., eds. Fundamentos da matéria orgânica do solo: Ecossistemas tropicais \& subtropicais. 2.ed. Porto Alegre, Metropole, 2008. v.1. p.443-461. 
SALL, J.; CREIGHTON, L. \& LEHMAN, A. JMP start statistics: A guide to statistics and data analysis using JMP and JMP IN software. 3.ed. Cary, Duxbury Press, 2005. 580p.

SIX, J.; S.D. FREY, R.K. THIET \& BATTEN, K.M. Bacterial and fungal contribution to carbon sequestration in agroecosystems. Soil Sci. Soc. Am. J., 70:555-569, 2006.

SIX, J.; FELLER,C.; DENEF,K.; OGLE,S.M.; SÁ,J.C.M. \& ALBRECHT, A. Soil organic matter, biota and aggregation in temperate and tropical soils-Effects of notillage. Agronomie, 22:755-775, 2002.

TISDALL, J.M. \& OADES, J.M. Organic matter and waterstable aggregates in soils. Soil Sci. Soc. Am. J., 33:141163, 1982.

TORMENA, C.A.; FRIEDRICH, R.; PINTRO, J.C.; COSTA, A.C.S. \& FIDALSKI, J. Propriedades físicas e taxa de estratificação de carbono orgânico num Latossolo Vermelho após dez anos sob dois sistemas de manejo. $\mathrm{R}$. Bras. Ci. Solo, 28:1023-1031, 2004.

TORMENA, C.A.; FIDALSKI, J. \& ROSSI JUNIOR, W. Resistência tênsil e friabilidade de um Latossolo sob diferentes sistemas de uso. R. Bras. Ci. Solo, 32:33-42, 2008a.
TORMENA, C.A.; ARAÚJO, M.A.; FIDALSKI, J.; IMHOFF, S. \& SILVA, A.P. Quantificação da resistência tênsil e da friabilidade de um Latossolo Vermelho distroférrico sob plantio direto. R. Bras. Ci. Solo, 32:943-952, 2008b.

UTOMO, W.H. \& DEXTER, A.R. Soil friability. J.Soil Sci., 32:203-213, 1981.

WATTS, C.W. \& DEXTER, A.R. The influence of organic matter in reducing the destabilization of soil by simulated tillage. Soil Tillage Res., 42:253-275, 1997.

WATTS, C.W. \& DEXTER, A.R. Soil friability: Theory, measurement and the effects of management and organic carbon content. Eur. J. Soil Sci., 49:73-84, 1998.

ZHANG, H. Organic matter incorporation affects on mechanical properties of soil aggregates. Soil Tillage Res., 31:263-175, 1994.

ZOTARELLI, L.; ALVES, B.J.R.; URQUIAGA, S.; BODDEY, R.M. \& SIX, J. Impact of tillage and crop rotation on light fraction and intra-aggregate soil organic matter in two Oxisols. Soil Tillage Res., 95:196-206, 2007. 
